\title{
SPECTRAL ANALYSES OF WC-TYPE CENTRAL STARS
}

\author{
W.-R. HAMANN, L. KOESTERKE \\ Institut für Theoretische Physik und Sternwarte der Universität, Kiel, FRG
}

Models have been developed in Kiel for massive (Pop. I) WR stars which account for multi-level non-LTE radiation transfer in spherically expanding atmospheres. The published (Koesterke et al. 1992) grid of models for WC composition (40\% helium, $60 \%$ carbon by mass) can be applied to low-mass stars as well by means of the scaling properties of WR spectra (Hamann et al. 1992) and allow a rough guess of the parameters, while individual calculations are necessary for a detailed analysis and the determination of the chemical composition.

We have analyzed the spectrum of the central star of NGC 6751. The obtained parameters - still somewhat preliminary - are given in the Table and compared to the previously analyzed CPN of Abell 78 (Werner \& Koesterke 1992) and Longmore 4 (cf. Werner et al., these proceedings). The luminosity has been adopted as $10^{3.7} L_{\odot}$ from evolutionary considerations for all three stars. The results reveal that the only essential difference between these three CPN concerns their mass-loss rate, which is exceptionally strong in NGC 6751. The Longmore 4 data refer to the state of high mass-loss observed fortuitously on Jan-27-1992, while in the quiet state Longmore 4 is of PG 1159 type with $\dot{M}$ being $10^{-8} M_{\odot)} \mathrm{yr}^{-1}$ or less. Despite of their mass-loss all three stars resemble PG 1159 stars, indicating a close relationship.

The remarkable existence of nitrogen in NGC 6751 and Abell 78 has also its counterpart in a PG 1159 star (namely PG 1144+005, cf. Werner \& Heber 1991); its explanation within the "born-again" scenario needs special assumptions on "mixing and burning" (cf. Schönberner \& Blöcker 1992).

\begin{tabular}{lccc}
\hline CPN & NGC 6751 & Abell 78 & Longmore 4 \\
\hline$T_{*} / \mathrm{kK}$ & 105 & 115 & 120 \\
$\log \dot{M} /\left(M_{\odot} \mathrm{yr}^{-1}\right)$ & -5.2 & -7.3 & -7.3 \\
$v_{\infty} /\left(\mathrm{km} \mathrm{s}^{-1}\right)$ & 2000 & 3700 & 4000 \\
helium [\% by mass] & 61.5 & 33 & 67 \\
carbon [\% by mass] & 27 & 50 & 25 \\
nitrogen [\% by mass] & 1.5 & 2 & 0 \\
oxygen [\% by mass] & 10 & 15 & 8 \\
\hline
\end{tabular}

Hamann W.-R., Leuenhagen U., Koesterke L., Wessolowski U.: 1992, A\&A 255, 200

Koesterke L., Hamann W.-R., Wessolowski U.: 1992, A\&A (in press)

Schönberner D., Blöcker T.: 1992, in Atmospheres of Early-type stars, U. Heber and C.S. Jeffery (eds.), Lecture Notes in Physics 401, Springer, p. 305

Werner K., Heber U.:1991, A\&A 247, 476

Werner K., Koesterke L.: 1992, in Atmospheres of Early-type stars, U. Heber and C.S. Jeffery (eds.), Lecture Notes in Physics 401, Springer, p. 288 